\title{
Effectiveness of Communication in the Management of the ASD Condition
}

\author{
Hissa Mohammed* \\ National Center for Cancer Care and Research, Qatar
}

*Corresponding author: Hissa Mohammed, National Center for Cancer Care and Research, Qatar, E-mail: hmohammed6787@gmail.com

Received: 18 Nov, 2017 | Accepted: 29 Jan, 2018 | Published: 02 Feb, 2018

Citation: Mohammed H (2018) Effectiveness of Communication in the Management of the ASD Condition. J Neurol Neurobiol 4(1): dx.doi. org/10.16966/2379-7150.146

Copyright: (c) 2018 Mohammed H. This is an open-access article distributed under the terms of the Creative Commons Attribution License, which permits unrestricted use, distribution, and reproduction in any medium, provided the original author and source are credited.

\section{Introduction}

Autism Spectrum Disorder (ASD) is a medical condition that negatively affects the mental health of patients. As a developmental disorder, ASD causes brain impairment, so afflicted individuals have weak communication skills and poor social interaction skills. Young patients with ASD also display repetitive behavior $s$ and have a low interest in engaging in social activities. Initial symptoms of the condition are recognized when a child is two years old. It is important for parents to seek early medical intervention from pediatricians in order to enhance a child's ability to function in society [1]. The purpose of the research study is to examine the benefits of effective communication in enhancing healthcare outcomes for patients with ASD. Proper communication between radiographers and pediatric patients with ASD is significant in order to minimize a patient's exposure to radiation.

\section{Literature Review}

ASD does not have a cure. However, therapy can be provided to enhance the communication skills and the behavioral and social interaction abilities of affected individuals. Speech therapy is important in improving the oral communication skills of affected children from the early stages of life. Occupational therapy aims at improving a child's competency in social interactions and in his/her ability to perform basic tasks, such as dressing or bathing. Moreover, it is important to provide educational support that addresses the special needs of learners who have ASD. One type of support entails providing extra lessons to learners who are slow in understanding concepts due to ASD [2].
Adults that are affected by ASD also had ASD symptoms during childhood. However, they may not have received a proper diagnosis or learned how to manage their condition. Proper diagnosis is important among adult patients with ASD because it enables them and their families to learn effective care strategies and receive appropriate support [3]. There are several autism-specific programs that enable affected patients to live independently and secure employment opportunities that are in line with their interests and abilities.

\section{Methodology}

The present research study aims to determine if effective communication between young ASD patients and healthcare practitioners is important in limiting the patient's exposure to radiation. Most young patients become anxious when they are receive care from healthcare workers they do not know. Therefore, providing proper care to young ASD patients requires a healthcare professional who is friendly and has good communication skills $[1,4]$. Successful imaging that has minimal radiation exposure occurs when radiographers maintain good communication with ASD young patients and their parents. The qualitative research process used in the present study had 10 respondents. Five of the 10 respondents were radiographers, and five were the mothers of young ASD patients.

A questionnaire was use to obtain data from the 10 respondents. The confidentiality of the data was ensured because the respondents did not indicate their names on the questionnaire they completed and returned. The data collected from the questionnaires was analyzed using STRATA software. The mothers provided information about the conduct of the young patients in various environments. They also provided information about the conditions that calm their children. The radiographers provided data about the problems that they experience when administering the imaging procedure to young patients. The intention of the data collection process was to provide radiographers with strategies to enhance the imaging process based on the mother's experiences of with their children. 


\section{Discussion}

Proper communication between radiographers and young ASD patients and their mothers is important in order to improve the quality of care. Proper communication reduces the children's anxiety levels; this improves their cooperation during the radiation process, which reduces the duration of their exposure to radiation. The communication skills of the healthcare practitioners can be improved in various ways. Training is an essential first step in enhancing communication with young patients. Radiographers and other healthcare staff should be enrolled in a learning program that improves their relationship with young patients and strengthens their ability to exchange information with young patients and their parents or caregivers. Teamwork is another important step in enhancing the communication abilities of healthcare staff. Radiographers should work together to determine the most effective communication strategy to use with young ASD patients and their parents or caregivers. It is important to manage ASD in the early stages of the condition. Young patients and their parents can also enroll in an autism support group [5]. The support group is important because it enhances the radiologists' ability to share information, which then improves the ability to manage a child's autism.

\section{Conclusion}

The result of the present research study shows the benefits of proper communication in the management of ASD in pediatric patients. Effective communication creates a relaxing care environment for patients with ASD, which enables radiographers to conduct the imaging procedure in the shortest amount of time possible, thereby reducing the patient's anxiety and their exposure to radiation.

\section{References}

1. Wong C, Odom S, Hume KA, Cox AW, Fettig A, et al. (2015) Evidence-based practices for children, youth, and young adults with autism spectrum disorder: A comprehensive review. J Autism Dev Disord 45: 1951-1966.

2. Brunsdon VE, Colvert E, Ames C, Garnett T, Gillan N, et al. (2015) Exploring the cognitive features in children with autism spectrum disorder, their co-twins, and typically developing children within a population-based sample. J Child Psychol Psychiatry 55: 893902.

3. Christensen DL, Bilder DA, Zahorodny W, Pettygrove S, Durkin MS, et al. (2016) Prevalence and characteristics of autism spectrum disorder among 4-year-old children in the autism and developmental disabilities monitoring network. J Dev Behav Pediatr 37: 1-8.

4. Developmental D.M.N.S.Y and 2010 Principal Investigators (2014) Prevalence of autism spectrum disorder among children aged 8 years-autism and developmental disabilities monitoring network, 11 sites, United States, 2010. MMWR Surveill Summ 63: 1-21.

5. Blumberg SJ, Bramlett MD, Kogan MD, Schieve LA, Jones JR, et al. (2013) Changes in prevalence of parent-reported autism spectrum disorder in school-aged US children: 2007 to 20112012. Natl Health Stat Report 20: 1-11. 